\title{
The Influence of Action Video Games on Attentional Functions Across Visual and Auditory Modalities
}

\author{
Xia Wu ${ }^{1,2,3,4}$, Ying Jiang ${ }^{2}$, Yunpeng Jiang ${ }^{1,2,3}$, Guodong Chen ${ }^{5}$, Ying Chen ${ }^{6}$ and \\ Xuejun Bai ${ }^{1,2,3 *}$
}

\begin{abstract}
'Key Research Base of Humanities and Social Sciences of the Ministry of Education, Academy of Psychology and Behavior, Tianjin Normal University, Tianjin, China, ${ }^{2}$ Faculty of Psychology, Tianjin Normal University, Tianjin, China, ${ }^{3}$ Tianjin Social Science Laboratory of Students' Mental Development and Learning, Tianjin, China, ${ }^{4}$ Institute of Psychology, Chinese Academy of Sciences, Beijing, China, ${ }^{5}$ Zhonghuan Information College, Tianjin University of Technology, Tianjin, China, ${ }^{6}$ Department of Social Psychology, Zhou Enlai School of Government, Nankai University, Tianjin, China
\end{abstract}

OPEN ACCESS

Edited by:

Sjoerd J. H. Ebisch, University of Studies G. d'Annunzio

Chieti and Pescara, Italy

Reviewed by:

Adriana L. Ruiz-Rizzo,

Ludwig Maximilian University

of Munich, Germany

Robert West

DePauw University, United States

${ }^{*}$ Correspondence:

Xuejun Bai

baixuejun@tjnu.edu.cn

Specialty section

This article was submitted to

Cognitive Science,

a section of the journal

Frontiers in Psychology

Received: 29 September 2020

Accepted: 06 May 2021

Published: 02 June 2021

Citation:

Wu X, Jiang Y, Jiang Y, Chen $G$,

Chen $Y$ and Bai $X$ (2021) The Influence of Action Video Games on Attentional Functions Across Visual

and Auditory Modalities.

Front. Psychol. 12:611778.

doi: 10.3389/fpsyg.2021.611778
Attention can help an individual efficiently find a specific target among multiple distractors and is proposed to consist of three functions: alerting, orienting, and executive control. Action video games (AVGs) have been shown to enhance attention. However, whether AVG can affect the attentional functions across different modalities remains to be determined. In the present study, a group of action video game players (AVGPs) and a group of non-action video game players (NAVGPS) selected by a video game usage questionnaire successively participated in two tasks, including an attention network task-visual version (ANT-V) and an attention network task-auditory version (ANT-A). The results indicated that AVGPs showed an advantage in orienting under the effects of conflicting stimuli (executive control) in both tasks, and NAVGPs may have a reduced ability to disengage when conflict occurs in visual task, suggesting that the AVGs can improve guidance toward targets and inhibition of distractors with the function of executive control. AVGPs also showed more correlations among attentional functions. Importantly, the alerting functions of AVGPs in visual and auditory tasks were significantly related, indicating that the experience of AVGs could help us to generate a supramodal alerting effect across visual and auditory modalities.

Keywords: action video games, visual and auditory modalities, attention network test, executive control, attentional functions

\section{INTRODUCTION}

Although some evidence has proven that playing violent video games is related to aggressive behaviors (Anderson et al., 2010; Prescott et al., 2018), the benefits of action video games (AVGs) on attention are one of the emerging fields for understanding their impact on child development (Granic et al., 2014), and researchers have further suggested that AVGs can influence mental health and education (Li and Tsai, 2013; Bavelier and Green, 2019). Attention refers to the process of selecting task-relevant stimuli and inhibiting task-irrelevant distractors, and it helps us to allocate the mental resources involved in a vast number of simultaneous inputs from visual, auditory and other sensory modalities. Relative to non-action video game players (NAVGPs), action video game players (AVGPs) are considered to have more attentional resources (Feng et al., 2007) and a better 
ability to accomplish selective attention in the visual modality (Green and Bavelier, 2003; Feng et al., 2007; Dye et al., 2009; Xiang and Hu, 2010; Hubert-Wallander et al., 2011), as well as greater phonological decoding speed in the auditory modality (Sandro and Sara, 2018). Although the experience of AVGs can improve probabilistic inference in both visual and auditory tasks (Green et al., 2010), whether it can influence attention across modalities remains to be revealed.

Attention is postulated to comprise a set of attentional functions, including an alerting function responsible for maintaining the state of response readiness to external warning stimuli, an orienting function responsible for selecting relevant information from numerous sensory inputs, and an executive control function responsible for detecting and resolving conflict among competing mental processes (Fan et al., 2002). Studies have found that AVGs can affect attentional functions. In terms of alerting, Donohue et al. (2010) found AVGPs to be good at determining the temporal sequence of multisensory stimuli. In terms of orienting, Hubert-Wallander et al. (2011) showed that AVGs could enhance the efficiency of selective visual attention. Franceschini et al. (2017) found that AVGs could improve the shifting of attention between visual and auditory modalities. In terms of executive control, Bavelier et al. (2012) and Krishnan et al. (2013) reported that AVGPs were better able to exclude task-irrelevant information. Bailey et al. (2010) and West et al. (2020) found that AVGs experience and training can reduce proactive cognitive control. However, the influence of AVGs on the interactions among attentional functions remain unclear.

Characterizing the supramodal and modality-specific mechanisms of the attentional functions could clarify their hierarchical structure. Specifically, executive control at a higher hierarchical level is considered to be responsible for coordinating mental computations and integrating information across modalities and has been proven to be supramodal. In contrast, alerting and orienting at a lower level are responsible for encoding sensory information, so they have been found to be modality-specific (Spagna et al., 2015, 2020). After the experience of quickly recognizing targets (e.g., in shooter games), responding to opponents (e.g., in fighting games) or changing formations (e.g., in sports games), the visual-auditory correlations of AVGPs may be altered in the modality-specific mechanisms of alerting and orienting.

In the present study, we investigated the influence of AVGs on the mechanisms and interactions of different attentional functions across visual and auditory modalities. By employing an attention network test (ANT, Fan et al., 2002), we can examine the efficiency of alerting, orienting and executive control simultaneously. AVGPs and NAVGPs were asked to successively complete visual and auditory versions of the ANT (Fan et al., 2009; Spagna et al., 2015) in a single session so that we could measure the within-subject correlations between attentional functions across visual and auditory tasks. The effects of AVGs on attention can be revealed by comparing AVGPs and NAVGPs with respect to the defined effects. The supramodal or modalityspecific mechanisms of attentional functions were examined by the correlations between the attentional effects in the visual and auditory modalities. We predicted that the function of executive control and the correlations between visual and auditory task performance would be enhanced in the AVGP group relative to the NAVGP group. In addition, a supramodal mechanism for each attentional function and their interactions was found in the AVGP group, indicating the influence of AVGs experience on the mechanism of attention.

\section{MATERIALS AND METHODS}

\section{Participants}

Sixty-eight adult volunteers were chosen based on a Video Game Usage Questionnaire and participated in this study. After excluding nine participants due to their low average accuracies $(<70 \%)$ in both experiments, the remaining 59 participants (31 males and 28 females; mean \pm standard deviation of age: $19.92 \pm 1.51$ years, range $18-24$ years) were divided into the AVGP group (21 males and 10 females; mean \pm standard deviation of age: $20.07 \pm 1.65$ years) and the NAVGP group (10 males and 18 females; mean \pm standard deviation of age: $19.75 \pm 1.33$ years). All participants were undergraduate students, and they were chosen in equal numbers from different grades and majors (arts, sciences, engineering). All participants were right-handed had normal or corrected-to-normal visual acuity and normal hearing. They signed informed consent before the experiment, and a certain remuneration was given to each after the experiment. They completed the visual (ANT-V) and auditory (ANT-A) versions of the ANT with a break of $10 \mathrm{~min}$, and the order of the tasks was balanced among the participants. The study was approved by the Ethics Committee of Tianjin Normal University.

The Video Game Usage Questionnaire was adapted from the Video Game Questionnaire (University of California, Berkeley database) and combined with assessment of current domestic use of games. The video games were classified into two types: action video games (AVGs), including action games (ACT, such as Devil May Cry, God of War, etc., Q9), first-person shooter games (FPS, such as CSGO, CrossFire, PUBG, etc., Q10), fighting games (such as: Street Fighter, The King of Fighters, etc., Q11), MOBA games (such as DOTA, League of Legends, Glory of Kings, etc., Q14), sports games (such as NBA, FIFA, Pro Evolution Soccer, etc., Q16), and real-time strategy games (RTS, such as StarCraft, Warcraft, etc., Q18); Non-action video games (NAVGs) includes strategy games, card games, puzzle games, etc (all the remaining questions). Referring to the research by Donohue et al. (2010), an AVGP is defined as a player who has played an average of more than $8 \mathrm{~h}$ of any type of action video game per week in the past 6 months; a NAVGP is defined as a player who has not played video games or has just played card games or puzzle games in the past 6 months.

\section{Attention Network Task-Visual Version (ANT-V)}

In the ANT-V (Figure 1), the stimuli were presented on a 15.6inch monitor $(1366 \times 768$ pixels, $60 \mathrm{~Hz})$, and the participants were required to look at the central fixation cross (angle $1^{\circ}$ ) throughout the experiment. Participants were instructed to 


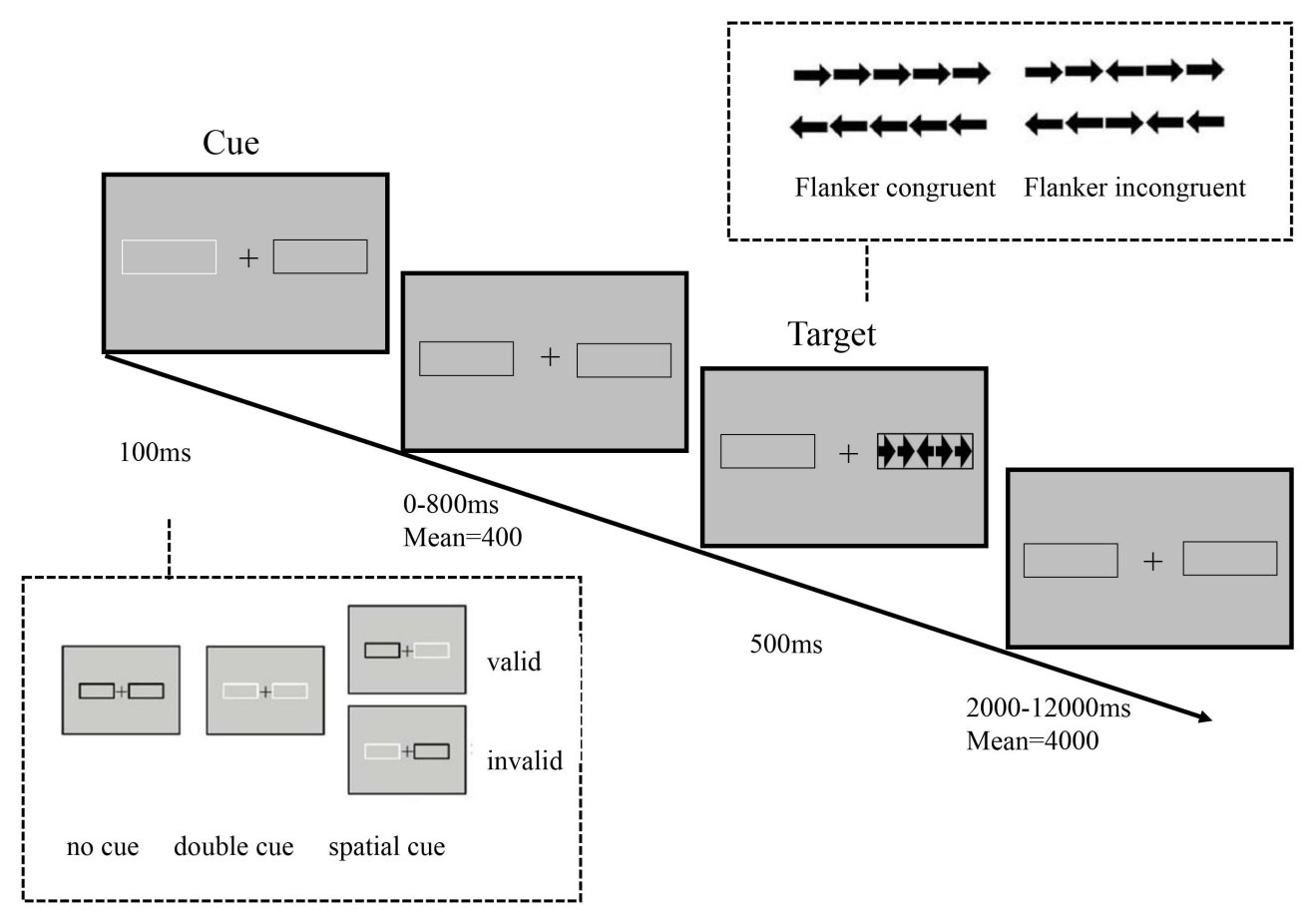

FIGURE 1 | Schematic of the visual attention network task (ANT-V).

respond to the direction of the central arrow (left or right) as quickly and accurately as possible by pressing with the left or right index finger. The target arrow was flanked by four surrounding flanker arrows pointing in the same direction (congruent condition) or in the opposite direction (incongruent condition) with equal probability. The arrows (each visual angle was $0.58^{\circ}$ with a gap of $0.06^{\circ}$ ) appeared in one of two black frames subtending $3.27^{\circ}$ to the left and right of central fixation. In each trial, a cue occurred for $100 \mathrm{~ms}$ with a randomized duration $(0,400$, or $800 \mathrm{~ms})$ before the appearance of arrows, with three possible conditions: (1) no cue: the screen remained unchanged; (2) double cue: both black frames were briefly changed from black to white; (3) spatial cue: only one of the two black frames changed, with $75 \%$ of the cues in the same location as the target arrow (valid cues) and $25 \%$ of the cues in the opposite location (invalid cues). The durations of precue fixation and posttarget fixation were $3000 \mathrm{~ms}$ and a randomized value between 2,000 and $12,000 \mathrm{~ms}$ (with a mean of 4,000 ms), respectively. The task included four blocks, each with 72 trials, for a total of 288 trials. Of the total trial number, there were 48 trials for the no cue condition, 48 trials for the double cue condition, 48 trials for the invalid cue condition, and 144 for the valid cue condition. The entire experiment last approximately $40 \mathrm{~min}$.

\section{Attention Network Task-Auditory Version (ANT-A)}

In the ANT-A (Figure 2), the stimuli were presented on a 15.6-inch monitor $(1366 \times 768$ pixels, $60 \mathrm{~Hz})$, and the participants were required to look at the central fixation cross (angle $1^{\circ}$ ) and wear headphones (frequency response from 20 to $20,000 \mathrm{~Hz}$ ) throughout the experiment. Participants were asked to indicate the duration (length) of a binaurally presented tone (short or long duration of either 30 or $150 \mathrm{~ms}$, respectively) as quickly and accurately as possible by pressing the index button. The target tone was followed by another binaurally presented tone as a flanker, and the flanker tone was either the same length as the target (congruent condition) or the other length (incongruent condition) with equal probability. The tones occurred at either high $(1,500 \mathrm{~Hz})$ or low $(1,000 \mathrm{~Hz})$ frequency. In each trial, a cue occurred for $115 \mathrm{~ms}$, with three possible conditions: (1) no cue: no tone prior to the target; (2) double cue: a mixed tone of 1,000 and $1,500 \mathrm{~Hz}$ occurred; (3) frequency cue: a $1,000-\mathrm{Hz}$ or a $1,500-\mathrm{Hz}$ tone occurred, with $75 \%$ of the cues at the same frequency as the target (valid cues) and $25 \%$ of the cues at the other frequency (invalid cues). Then a blank screen was presented for $675 \mathrm{~ms}$ before the onset of the target tone. The durations of precue fixation and posttarget fixation were 3,000 ms and a random value between 2,000 and $12,000 \mathrm{~ms}$ (with a mean of $4,000 \mathrm{~ms}$ ), respectively. The operational definitions of the attentional effects and the experimental design were the same as those in the ANT-V.

\section{The Experimental Design and the Operational Definitions of the Attentional Effects}

The factorial design was 2 (group: AVGP group, NAVGP group) $\times 4$ (cue condition: no cue, double cue, valid cue, 


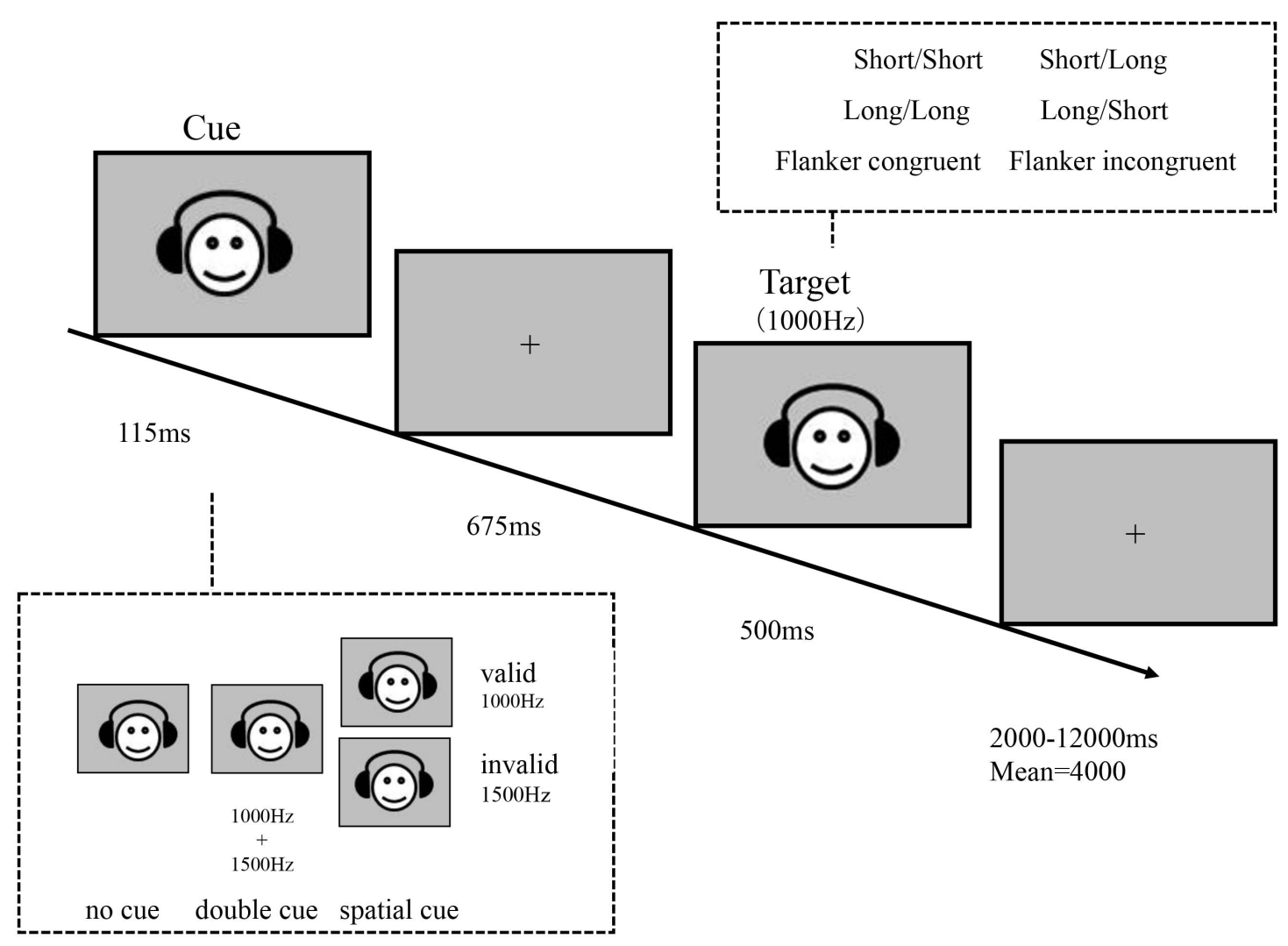

FIGURE 2 | Schematic of the auditory attention network task (ANT-A).

invalid cue) $\times 2$ (target condition: congruent, incongruent) Error rate (ER) and reaction times (RTs) were calculated separately for each condition. RTs with incorrect answers or exceeding three standard deviations (SDs) of the mean of each condition were excluded from further analyses. The formulas used to define the attentional effects and interactions are listed in Table 1. All attentional effects and interactions were calculated to explore any possible effect of AVGs on attention. To investigate the effects of AVGs on attention, one-way ANCOVA was performed for the RTs and the error rate of each defined attentional effect and interaction with the group (AVGP and NAVGP) as the between-subject variable and gender as a covariate (following the methods of Spagna et al., 2015, 2018). A significant main effect of group would reveal a significant effect of AVGs. For each defined interaction with a significant effect, a further planned mixed ANCOVA with group (AVGP, NAVGP, between-subject variable) and the corresponding cue conditions (within-subject variables) was conducted to investigate the simple effects underlying this interaction. Moreover, to investigate the relationships among the attentional effects, Pearson's correlation analyses were conducted for each attentional effect and interaction. FDR correction for multiple comparisons was employed. The $p$-values for each analysis were adjusted for multiple comparisons to a $P_{\mathrm{FDR}}<0.05$. Correlations were compared by using psychometrica.de ${ }^{1}$. SPSS software package (SPSS Inc. Chertsey, United Kingdom, version 22.0) was used to analyze the data.

\section{RESULTS}

\section{Differences in Attentional Effects Between AVGPs and NAVGPs in the ANT-V}

The error rate and RTs for correct responses $(M \pm S D)$ of different groups for each condition and attentional effect are shown in Table 2 and Figure 3, respectively.

One-way ANCOVA was conducted for the error rate of each attentional effect and interaction. The results showed that two groups were not significantly different in the average performance in all conditions (the average performance on all conditions) $[F(1,56)=0.313, p=0.578]$, alerting $[F(1,56)$ $=0.003, p=0.954]$, disengaging $[F(1,56)=0.526, p=$ $0.471]$, orienting $[F(1,56)=1.239, p=0.270]$, validity $[F(1,56)=$ $1.837, p=0.181]$, conflict $[F(1,56)=0.554, p=0.460]$, alerting by conflict $[F(1,56)=0.966, p=0.330]$, disengaging by conflict $[F(1,56)=0.746, p=0.391]$, orienting by conflict $[F(1,56)=$

\footnotetext{
${ }^{1}$ https://www.psychometrica.de/correlation.html
} 
TABLE 1 | Operational definitions of the attentional effects and interactions.

\begin{tabular}{|c|c|c|c|}
\hline & Testing condition & Minus & Reference condition \\
\hline \multicolumn{4}{|l|}{ Attentional effects } \\
\hline Alerting & No cue & & Double cue \\
\hline Disengaging & Invalid cue & & Double cue \\
\hline Orienting & Double cue & & Valid cue \\
\hline Validity & Invalid cue & & Valid cue \\
\hline Conflict & Incongruent cue & & Congruent cue \\
\hline \multicolumn{4}{|l|}{ Interactions } \\
\hline Alerting by conflict & No cue, incongruent minus no cue, congruent & & Double cue, incongruent minus double cue, congruent \\
\hline Disengaging by conflict & Invalid cue, incongruent minus invalid cue, congruent & & Double cue, incongruent minus double cue, congruent \\
\hline Orienting by conflict & Double cue, incongruent minus double cue, congruent & & Valid cue, incongruent minus valid cue, congruent \\
\hline Validity by conflict & Invalid cue, incongruent minus invalid cue, congruent & & Valid cue, incongruent minus valid cue, congruent \\
\hline
\end{tabular}

The conflict effect reflects the function of executive control.

TABLE 2 | Mean (SD) reaction time (RTs, ms) and error rate (ER, \%) for each condition in the ANT-V.

\begin{tabular}{|c|c|c|c|c|c|}
\hline Group & & Double cue & No cue & Valid cue & Invalid cue \\
\hline \multicolumn{6}{|l|}{ RT } \\
\hline \multirow[t]{2}{*}{ AVGP } & Congruent & $536(53.00)$ & $573(50.28)$ & 503 (52.95) & 589 (53.68) \\
\hline & Incongruent & 684 (62.33) & 725 (73.84) & $601(67.45)$ & 753 (84.58) \\
\hline \multirow[t]{2}{*}{ NAVGP } & Congruent & $542(55.08)$ & $576(65.65)$ & $500(51.37)$ & $581(58.16)$ \\
\hline & Incongruent & 687 (89.26) & 730 (89.49) & 625 (86.14) & $774(99.42)$ \\
\hline \multicolumn{6}{|c|}{ Error rate } \\
\hline \multirow[t]{2}{*}{ AVGP } & Congruent & $0.40(1.25)$ & $0.67(1.56)$ & 1.03 (1.76) & 1.08 (2.63) \\
\hline & Incongruent & 11.56 (12.06) & $13.71(13.23)$ & 7.26 (7.88) & 17.07 (13.83) \\
\hline \multirow[t]{2}{*}{ NAVGP } & Congruent & $0.15(0.79)$ & 1.19 (2.23) & $0.74(1.22)$ & $0.45(1.73)$ \\
\hline & Incongruent & $11.46(12.71)$ & $12.65(16.14)$ & 6.55 (8.96) & $19.64(19.51)$ \\
\hline
\end{tabular}

1.232, $p=0.272]$, nor validity by conflict $[F(1,56)=2.350, p=$ $0.131]$.

One-way ANCOVA was conducted for the RTs of each attentional effect and interaction. The results showed that two groups were not significantly different in the average performance in all conditions $[F(1,56)=0$. 044, $p=$ $0.835]$, alerting $[F(1,56)=0.034, p=0.854]$, disengaging $[F(1,56)$ $=0.014, p=0.905]$, orienting $[F(1,56)=1.802, p=0.185]$, validity $[F(1,56)=1.068, p=0.306]$, conflict $[F(1,56)=1.379, p=$ $0.245]$, alerting by conflict $[F(1,56)=0.654, p=0.422]$, nor validity by conflict $[F(1,56)=0.006, p=0.941]$.

However, a marginally significant difference in RTs between the two groups for disengaging by conflict $[F(1,56)=3.734, p$ $\left.=0.058, \omega^{2}=0.044\right]$, suggesting that the effects tend to be lower for AVGPs (15.52 \pm 51.68) than for NAVGPs $(47.49 \pm 57.09)^{2}$, although the effect size was relatively small. To explore the separate effect on disengaging by conflict in different groups and the underlying simple effect in this interaction, a further mixed ANCOVA for the conflict effects with group (AVGP, NAVGP) and cue condition (invalid cue, double cue) was performed. The simple effect analysis of the interaction showed no significant difference in the reaction to conflict between two the cue conditions in AVGPs $[F(1,56)=2.820, p=0.099]$, but

\footnotetext{
${ }^{2}$ The effects were calculated by subtraction, so that they reflect the relative magnitude of values rather than the absolute values of RTs.
}

the reaction to conflicting stimuli in the context of invalid cues was significantly lower than that with double cues in NAVGPs $[F(1,56)=18.702, p<0.001]$ (Figure 4A), suggesting that NAVGPs may have reduced ability to disengage attention when conflict occurs. There was no significant difference in the conflict effect between the two groups, either for the invalid cue $[F(1,56)$ $=2.475, p=0.121]$ or the double cue $[F(1,56)=0.017, p=$ 0.898] (Figure 4B).

In addition, a significant difference in RTs between the two groups for orienting by conflict $[F(1,56)=7.081, p=0.010$, $\left.\omega^{2}=0.094\right]$ showed that the effects were significantly higher for AVGPs (50.39 \pm 40.21$)$ than for NAVGPs (20.35 \pm 41.09$)$. To explore the separate effect on orienting in the context of conflicting stimuli in different groups and the underlying simple effect in this interaction, a further mixed ANCOVA for the conflict effects with group (AVGP, NAVGP) and cue condition (double cue, valid cue) was performed. The simple effect analysis of the interaction showed that RTs for conflicting stimuli in the context of double cues was significantly higher than that in the context of valid cues in both AVGPs $[F(1,56)=44.420, p<0.001]$ and NAVGPs $[F(1,56)=6.517, p=0.013]$ (Figure 4C), suggesting that both AVGPs and NAVGPs retained the ability to orient when conflict occurred. There was no significant difference in the conflict effect between the two groups in the double cue condition $[F(1,56)=0.017, p=0.898]$, but the conflict effect was significantly higher for AVGPs than for NAVGPs in the valid cue 


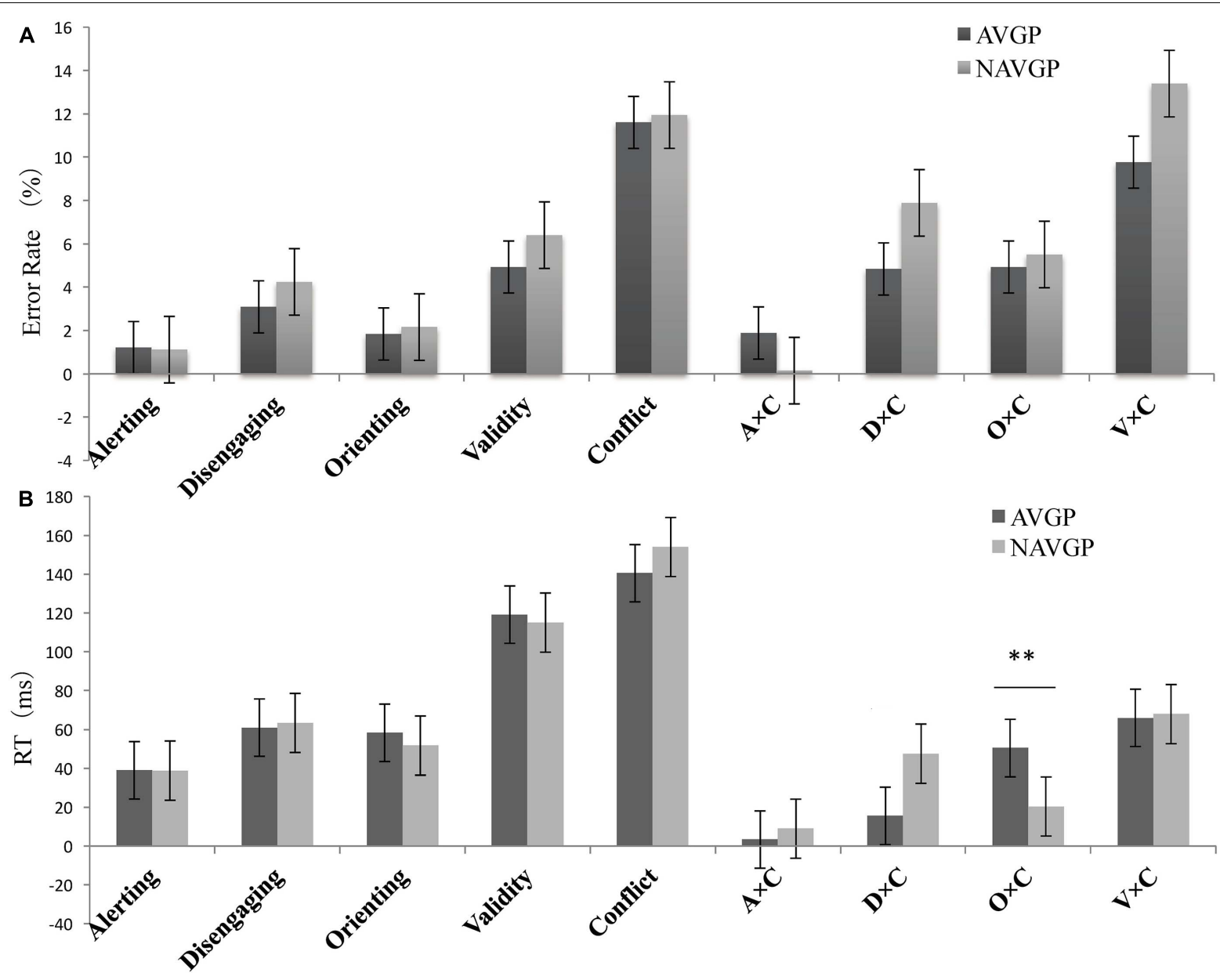

FIGURE 3 | (A) Error rate (ER, \%) and (B) Reaction times (RTs) as a function of the attentional effects of different groups in the ANT-V. Error bars represent the standard error of the mean. $\mathrm{A} \times \mathrm{C}$, alerting by conflict; $\mathrm{D} \times \mathrm{C}$, disengaging by conflict; $\mathrm{O} \times \mathrm{C}$, orienting by conflict; $\mathrm{V} \times \mathrm{C}$, validity by conflict. $* * p<0.01$.

condition $[F(1,56)=4.713, p=0.034]$ (Figure 4D), indicating that AVGPs possess increased ability to resolve conflict with the valid orientation.

\section{The Correlations Between Attentional Effects for AVGPs and NAVGPs in the ANT-V}

The coefficients of correlation between RTs to different attentional effects for AVGPs and NAVGPs are shown in Table 3. The AVGP group showed a significant positive correlation between disengaging and the interaction of validity and conflict $(r=0.655, p=0.001)$ and a significant positive correlation between conflict and the interaction of validity and conflict ( $r=0.521, p=0.015)$. No such correlations were found for NAVGPs $(r=0.444, p=0.052 ; r=0.099, p=0.757)$. NAVGPs showed a significant negative correlation between alerting and orienting $(r=-0.662, p=0.001)$, a significant positive correlation between alerting and conflict $(r=0.472, p=0.037)$, disengaging and conflict $(r=0.471, p=0.037)$, orienting and validity $(r=0.520, p=0.026)$, orienting and the interaction of validity and conflict $(r=0.493, p=0.035)$, and validity and the interaction of disengaging and conflict $(r=0.601, p=0.005)$. However, no such correlations were found for AVGPs $(r=-0.438, p=0.052$; $r=0.227, p=0.340 ; r=0.422, p=0.058 ; r=0.391, p=0.088$; $r=0.010, p=0.980 ; r=0.279, p=0.231$, respectively).

The results and corresponding $p$-value from comparing the correlation coefficients of the attentional effects in AVGPs to those in NAVGPs are shown in Table 4. Relative to NAVGPs, AVGPs exhibited higher correlations between the average performance in all conditions and the interaction between alerting and conflict $(z=2.990, p=0.001)$, alerting and the interaction of validity and conflict $(z=1.957, p=0.025)$, and conflict and the interaction of validity and conflict $(z=1.740$, $p=0.041)$. However, relative to AVGPs, NAVGPs showed higher correlations between the average performance in all conditions and orienting $(z=-1.791, p=0.037)$, orienting and the interaction of disengaging and conflict $(z=-2.076, p=0.019)$ and orienting and the interaction of validity and conflict $(z=-1.926, p=0.027)$. The results described above showed that, in the visual modality, the correlation between alerting and conflict was stronger for AVGPs, while the correlations between 


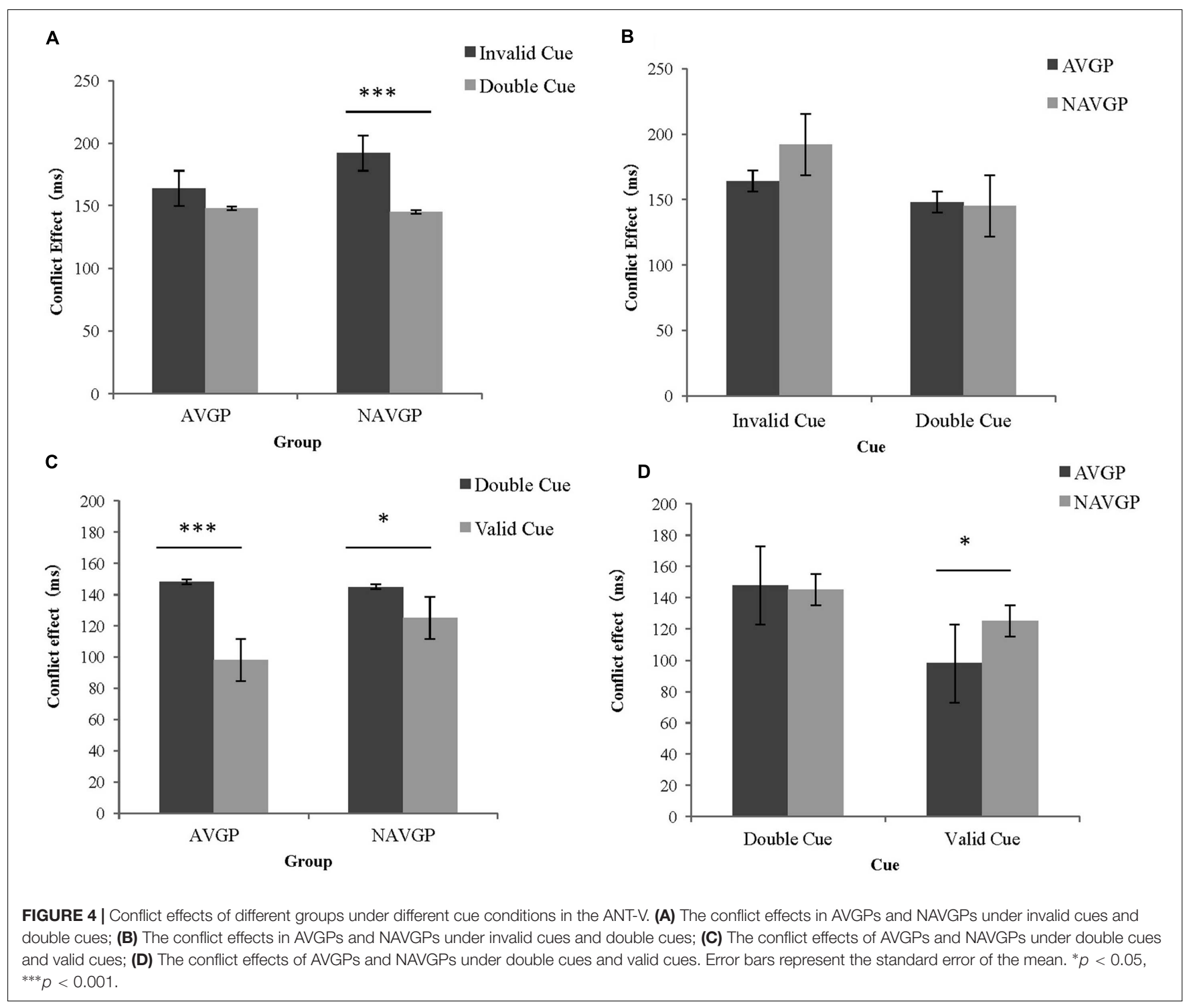

the functions of orienting (including the effects of validity, orienting, disengaging) and conflict were tighter for NAVGPs.

\section{Differences in Attentional Effects Between AVGPs and NAVGPs in the ANT-A}

Error rate and RTs for correct responses $(M \pm S D)$ of different groups for each condition and attentional effect are shown in Table 5 and Figure 5, respectively.

One-way ANCOVA was conducted for the error rate of each attentional effect and interaction. The results showed that two groups were not significantly different in the average performance in all conditions $[F(1,56)=0.021, p=0.885]$, alerting $[F(1,56)=1.203, p=0.277]$, disengaging $[F(1,56)=$ $0.010, p=0.921]$, orienting $[F(1,56)=0.027, p=0.870]$, validity $[F(1,56)=0.057, p=0.811]$, conflict $[F(1,56)=0.834, p=0.365]$, alerting by conflict $[F(1,56)=0.670, p=0.417]$, disengaging by conflict $[F(1,56)=0.180, p=0.673]$, orienting by conflict $[F(1,56)$ $=0.426, p=0.517]$, nor validity by conflict $[F(1,56)=0.006, p=$ $0.939]$.

One-way ANCOVA was conducted for the RTs of each attentional effect and interaction. The results showed that two groups were not significantly different in the average performance in all conditions $[F(1,56)=0.149, p=0.701]$, alerting $[F(1,56)=0.012, p=0.912]$, disengaging $[F(1,56)=$ $1.369, p=0.247]$, orienting $[F(1,56)=0.037, p=0.849]$, validity $[F(1,56)=1.404, p=0.241]$, conflict $[F(1,56)=0.010, p=0.919]$, alerting by conflict $[F(1,56)=0.519, p=0.474]$, disengaging by conflict $[F(1,56)=1.219, p=0.274]$, nor validity by conflict $[F(1,56)=0.889, p=0.350]$.

However, a significant difference in RTs between two groups was found in the interaction between orienting and conflict $\left[F(1,56)=6.466, p=0.014, \omega^{2}=0.084\right]$, showing that the effects were significantly higher for AVGPs $(33.59 \pm 69.21)$ than for NAVGPs $(-18.85 \pm 71.25)$. To explore the separate effects 
TABLE 3 | Correlation coefficients for the attentional effects in different groups in the ANT-V.

\begin{tabular}{|c|c|c|c|c|c|c|c|c|c|c|}
\hline Group & & Overall & Alerting & Disengaging & Orienting & Validity & Conflict & $A \times C$ & $D \times C$ & $O \times C$ \\
\hline \multirow[t]{9}{*}{ AVGP } & Alerting & 0.190 & & & & & & & & \\
\hline & Disengaging & 0.431 & $0.474^{\star}$ & & & & & & & \\
\hline & Orienting & -0.329 & -0.438 & -0.227 & & & & & & \\
\hline & Validity & 0.208 & 0.183 & $0.808^{\star \star \star}$ & 0.391 & & & & & \\
\hline & Conflict & $0.515^{\star}$ & 0.227 & 0.422 & -0.112 & 0.331 & & & & \\
\hline & $A \times C$ & 0.334 & 0.092 & 0.156 & -0.152 & 0.056 & 0.209 & & & \\
\hline & $\mathrm{D} \times \mathrm{C}$ & 0.340 & 0.271 & 0.439 & -0.223 & 0.279 & 0.180 & $0.593^{\star \star}$ & & \\
\hline & $\mathrm{O} \times \mathrm{C}$ & -0.088 & -0.005 & 0.134 & 0.298 & 0.306 & 0.324 & $-0.563^{\star \star}$ & $-0.591^{\star \star}$ & \\
\hline & $\mathrm{V} \times \mathrm{C}$ & 0.327 & 0.322 & $0.655^{\star \star}$ & 0.010 & $0.625^{\star \star}$ & $0.521^{*}$ & 0.187 & $0.652^{\star \star}$ & 0.226 \\
\hline \multirow[t]{9}{*}{ NAVGP } & Alerting & 0.255 & & & & & & & & \\
\hline & Disengaging & 0.181 & $0.478^{\star}$ & & & & & & & \\
\hline & Orienting & 0.149 & $-0.662^{\star \star}$ & -0.239 & & & & & & \\
\hline & Validity & 0.268 & -0.063 & $0.705^{\star \star \star}$ & $0.520^{\star}$ & & & & & \\
\hline & Conflict & $0.630^{\star \star}$ & $0.472^{*}$ & $0.471^{*}$ & -0.235 & 0.243 & & & & \\
\hline & $A \times C$ & -0.442 & -0.142 & 0.125 & -0.011 & 0.102 & -0.076 & & & \\
\hline & $\mathrm{D} \times \mathrm{C}$ & -0.097 & -0.174 & 0.408 & 0.331 & $0.601^{\star \star}$ & 0.117 & $0.495^{\star}$ & & \\
\hline & $\mathrm{O} \times \mathrm{C}$ & 0.196 & -0.015 & -0.003 & 0.165 & 0.118 & -0.037 & $-0.586^{\star \star}$ & $-0.475^{\star}$ & \\
\hline & $\mathrm{V} \times \mathrm{C}$ & 0.048 & -0.202 & 0.444 & $0.493^{\star}$ & $0.751^{\star \star \star}$ & 0.099 & 0.081 & $0.721^{\star \star \star}$ & 0.268 \\
\hline
\end{tabular}

Overall, the average performance over all conditions; $A \times C$, alerting by conflict; $O \times C$, orienting by conflict; $V \times C$, validity by conflict. ${ }^{*} p<0.05,{ }^{* *} p<0.01,{ }^{* * *} p<0.001$.

TABLE 4 | Comparison of the correlation coefficients for the attentional effects in different groups in the ANT-V.

\begin{tabular}{|c|c|c|c|c|c|c|c|c|c|}
\hline & Overall & Alerting & Disengaging & Orienting & Validity & Conflict & $A \times C$ & $D \times C$ & $\mathrm{O} \times \mathrm{C}$ \\
\hline Alerting & -0.248 & & & & & & & & \\
\hline Disengaging & 1.011 & -0.019 & & & & & & & \\
\hline Orienting & $-1.791^{\star}$ & 1.190 & 0.049 & & & & & & \\
\hline Validity & -0.234 & 0.901 & 0.882 & -0.591 & & & & & \\
\hline Conflict & -0.623 & -1.023 & -0.226 & 0.462 & 0.347 & & & & \\
\hline$A \times C$ & $2.990^{\star \star}$ & 0.853 & 0.116 & -0.518 & -0.170 & 1.047 & & & \\
\hline $\mathrm{D} \times \mathrm{C}$ & 1.639 & 1.646 & 0.136 & $-2.076^{\star}$ & -1.481 & 0.232 & 0.506 & & \\
\hline $\mathrm{O} \times \mathrm{C}$ & -1.041 & 0.036 & 0.500 & 0.509 & 0.719 & 1.358 & 0.123 & -0.591 & \\
\hline$V \times C$ & 1.061 & $1.957^{\star}$ & 1.115 & $-1.926^{\star}$ & -0.878 & $1.740^{\star}$ & 0.394 & -0.470 & -0.163 \\
\hline
\end{tabular}

Overall, the average performance over all conditions; $A \times C$, alerting by conflict; $O \times C$, orienting by conflict; $V \times C$, validity by conflict. ${ }^{\star} p<0.05,{ }^{* *} p<0.01$.

TABLE 5 | Mean (SD) reaction time (RTs, ms) and error rate (ER, \%) for each condition in the ANT-A.

\begin{tabular}{|c|c|c|c|c|c|}
\hline Group & & Double cue & No cue & Valid cue & Invalid cue \\
\hline \multicolumn{6}{|l|}{ RT } \\
\hline \multirow[t]{2}{*}{ AVGP } & Congruent & 753 (119.29) & 955 (123.86) & $750(117.17)$ & 773 (106.34) \\
\hline & Incongruent & 850 (176.26) & 1106 (162.67) & 813 (150.72) & $844(160.25)$ \\
\hline \multirow[t]{2}{*}{ NAVGP } & Congruent & 756 (124.90) & 939 (108.53) & 726.(118.95) & 772 (130.95) \\
\hline & Incongruent & 826 (171.93) & 1089 (172.47) & 815 (180.03) & 840 (189.56) \\
\hline \multicolumn{6}{|c|}{ Error rate } \\
\hline \multirow[t]{2}{*}{ AVGP } & Congruent & $6.45(6.53)$ & $13.44(10.41)$ & $5.82(4.10)$ & $5.78(6.60)$ \\
\hline & Incongruent & $15.32(12.14)$ & 38.58 (18.63) & $14.87(9.50)$ & 15.73 (13.55) \\
\hline \multirow[t]{2}{*}{ NAVGP } & Congruent & $5.21(6.07)$ & 9.67 (9.49) & $5.21(4.56)$ & $5.36(6.20)$ \\
\hline & Incongruent & 18.75 (12.65) & 37.35 (13.86) & $16.72(9.74)$ & $18.60(16.06)$ \\
\hline
\end{tabular}

of orienting on conflict in different groups and the underlying simple effect in this interaction, a further mixed ANCOVA for the conflict effects with group (AVGP, NAVGP) and cue condition (double cue, valid cue) was performed. Further simple effect analysis of the interaction showed that the RTs for conflicting stimuli in context of double cues was significantly higher than that in the context of valid cues in AVGPs $[F(1,56)=6.094, p=$ $0.017]$, but no such difference was found for NAVGPs $[F(1,56)$ $=1.580, p=0.214$ ] (Figure 6A), suggesting that AVGPs had increased ability to orient when conflict occurred. There were no significant differences in the conflict effect between the two groups in the context of double cues $[F(1,56)=0.936, p=$ 


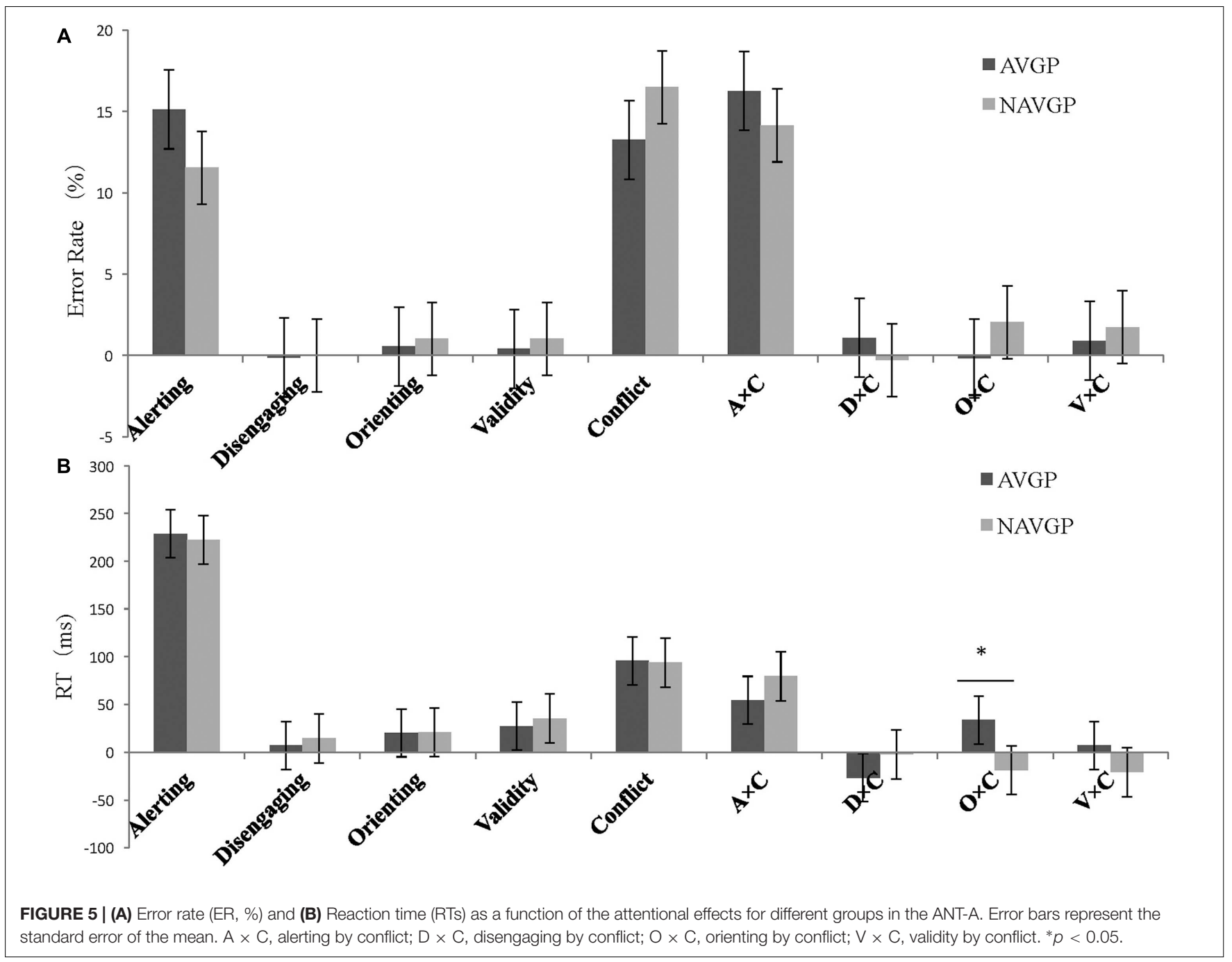

$0.338]$ or in the context of valid cues $[F(1,56)=1.905, p=$ 0.173] (Figure 6B).

\section{The Correlations Between Attentional Effects in AVGPs and NAVGPs in the \\ ANT-A}

The coefficients of correlation in RTs for different attentional effects in AVGPs and NAVGPs are shown in Table 6. AVGPs showed a significant negative correlation between alerting and orienting $(r=-0.481, p=0.031)$, orienting and the interaction between disengaging and conflict $(r=-0.485, p=0.031)$, a significant positive correlation between disengaging and the interaction between disengaging and conflict $(r=0.475, p=$ $0.031)$, disengaging and orienting $(r=0.509, p=0.022)$, and the interaction between alerting and conflict and the interaction between disengaging and conflict $(r=0.517, p=0.022)$. No such correlations were found for NAVGPs $(r=-0.078, p=0.866 ; r=$ $0.163, p=0.596 ; r=0.281, p=0.405 ; r=-0.469, p=0.080 ; r=$ $0.266, p=0.424$, respectively).
The results and corresponding $p$-value from comparisons of the correlation coefficients of the attentional effects in AVGPs to those in NAVGPs are shown in Table 7. Compared to NAVGPs, AVGPs exhibited higher correlations between average performance in all groups and interaction of orienting and conflict $(z=2.097, p=0.018)$, between alerting and interaction of validity and conflict $(z=1.890, p=0.029)$, and between orienting and interaction of disengaging and conflict $(z=-2.523, p=$ 0.006). The results described above showed that, in the auditory modality, the correlations between attentional functions were stronger for AVGPs than for NAVGPs.

\section{The Correlations Between RTs for Visual and Auditory Modalities for Attentional Effects in AVGPs and NAVGPs}

The coefficients of correlation between RTs for ANT-V and ANTA tasks for attentional effects in different groups are shown in Table 8. There were significant positive correlations between the RTs of the two tasks averaged over all conditions in AVGPs $(r=$ $0.392, p=0.029)$ and NAVGPs $(r=0.417, p=0.027)$. Importantly, 
A

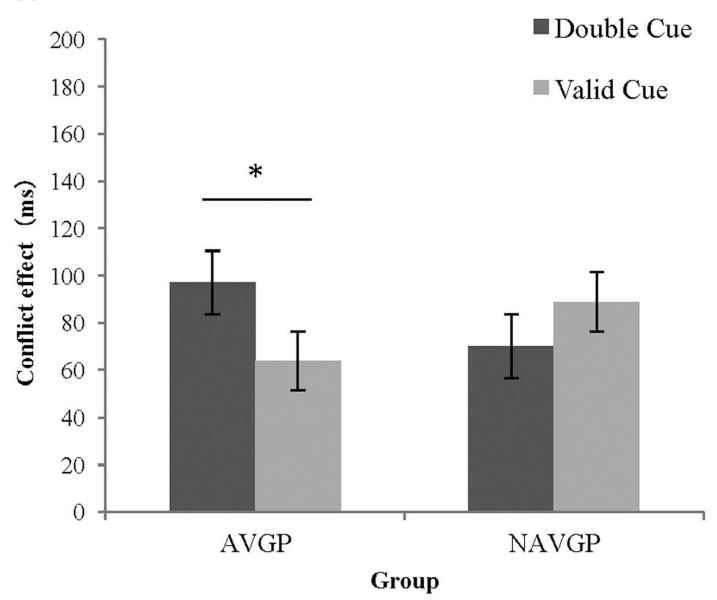

B

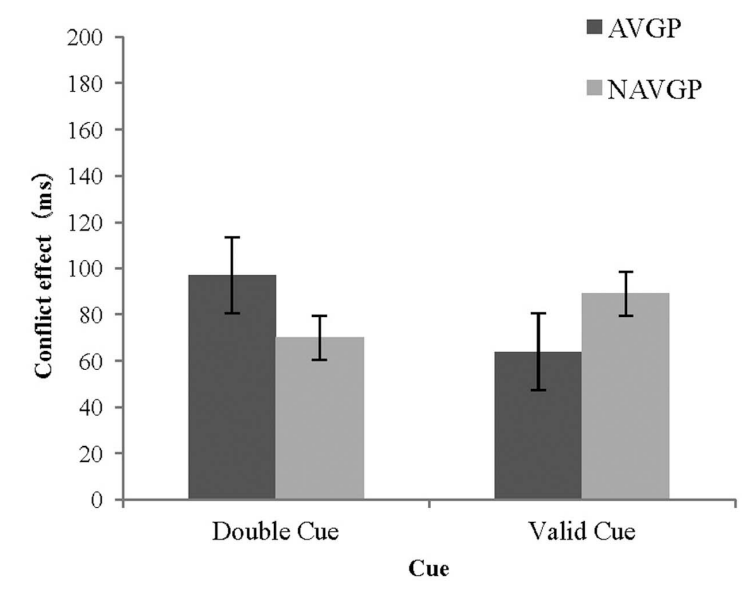

FIGURE 6 | Conflict effects of different groups under different cue conditions in the ANT-A (A) The conflict effects in AVGPs and NAVGPs under double cues and valid cues; (B) The conflict effects in AVGPs and NAVGPs under double cues and valid cues. Error bars represent the standard error of the mean. ${ }^{*} p<0.05$.

TABLE 6 | Correlation coefficients for the attentional effects in different groups in the ANT-A.

\begin{tabular}{|c|c|c|c|c|c|c|c|c|c|c|}
\hline Group & & Overall & Alerting & Disengaging & Orienting & Validity & Conflict & $A \times C$ & $D \times C$ & $O \times C$ \\
\hline \multirow[t]{9}{*}{ AVGP } & Alerting & -0.160 & & & & & & & & \\
\hline & Disengaging & -0.314 & 0.409 & & & & & & & \\
\hline & Orienting & 0.294 & $-0.481^{*}$ & $-0.509^{*}$ & & & & & & \\
\hline & Validity & -0.145 & 0.115 & $0.774^{\star \star \star}$ & 0.151 & & & & & \\
\hline & Conflict & $0.724^{\star \star \star}$ & -0.181 & -0.142 & 0.285 & 0.046 & & & & \\
\hline & $A \times C$ & -0.197 & 0.439 & 0.281 & -0.326 & 0.083 & -0.170 & & & \\
\hline & $\mathrm{D} \times \mathrm{C}$ & -0.028 & 0.303 & $0.475^{\star}$ & $-0.485^{\star}$ & 0.189 & 0.114 & $0.517^{\star}$ & & \\
\hline & $\mathrm{O} \times \mathrm{C}$ & 0.313 & -0.022 & -0.194 & 0.341 & 0.027 & 0.289 & $-0.610^{\star \star}$ & $-0.545^{\star}$ & \\
\hline & $\mathrm{V} \times \mathrm{C}$ & 0.225 & 0.338 & 0.397 & -0.289 & 0.244 & 0.371 & 0.104 & $0.724^{\star \star \star}$ & 0.184 \\
\hline \multirow[t]{9}{*}{ NAVGP } & Alerting & -0.246 & & & & & & & & \\
\hline & Disengaging & 0.223 & 0.451 & & & & & & & \\
\hline & Orienting & -0.098 & -0.078 & -0.469 & & & & & & \\
\hline & Validity & 0.191 & 0.461 & $0.839^{\star \star \star}$ & 0.088 & & & & & \\
\hline & Conflict & $0.784^{\star \star \star}$ & -0.453 & -0.017 & 0.002 & -0.017 & & & & \\
\hline & $A \times C$ & 0.306 & 0.173 & 0.246 & -0.162 & 0.177 & 0.277 & & & \\
\hline & $\mathrm{D} \times \mathrm{C}$ & 0.173 & -0.128 & 0.281 & 0.163 & 0.417 & 0.030 & 0.266 & & \\
\hline & $\mathrm{O} \times \mathrm{C}$ & -0.247 & -0.020 & -0.307 & 0.068 & -0.304 & -0.105 & $-0.631^{\star \star}$ & $-0.563^{\star}$ & \\
\hline & $\mathrm{V} \times \mathrm{C}$ & -0.033 & -0.167 & 0.037 & 0.252 & 0.197 & -0.064 & -0.285 & $0.629^{\star \star}$ & 0.289 \\
\hline
\end{tabular}

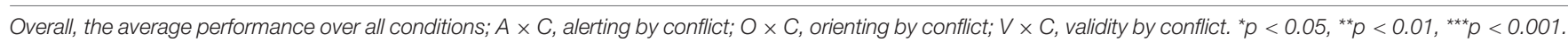

a significant positive correlation was observed between alerting in the two tasks in AVGPs ( $r=0.396, p=0.027)$, whereas there was no significant correlation in NAVGPs $(r=-0.339, p=0.078)$. Comparison of correlation coefficients for attentional effects in different modalities between AVGPs and NAVGPs showed that AVGPs had better ability to generate a supramodal alerting function $(z=2.805, p=0.003)$.

\section{DISCUSSION}

In the present study, in a within-subject design, we investigated the influence of action video games (AVGs) on the attentional functions across visual and auditory modalities by simultaneously testing the effects on alerting, orienting and executive control. Our results found that, relative to the results for non-action video game players (NAVGPs), action video game players (AVGPs) showed a significant interaction between orienting and conflict (executive control) functions in both visual and auditory modalities, suggesting that AVGPs have an advantage in orienting when conflict occurs. Furthermore, AVGPs also exhibited more correlations among the three attentional functions, indicating their benefits for binding attentional processing and for efficiency of target identification. Interestingly, a significant correlation between the performance of visual and auditory tasks was found in the alerting effect in AVGPs, 
TABLE 7 | Comparison of the correlation coefficients for the attentional effects in different groups in the ANT-A.

\begin{tabular}{|c|c|c|c|c|c|c|c|c|c|}
\hline & Overall & Alerting & Disengaging & Orienting & Validity & Conflict & $A \times C$ & $D \times C$ & $O \times C$ \\
\hline Alerting & 0.328 & & & & & & & & \\
\hline Disengaging & $-2.008^{\star}$ & -0.190 & & & & & & & \\
\hline Orienting & 1.457 & -1.623 & -0.193 & & & & & & \\
\hline Validity & -1.235 & -1.390 & -0.675 & 0.231 & & & & & \\
\hline Conflict & -0.508 & 1.110 & -0.460 & 1.057 & 0.232 & & & & \\
\hline$A \times C$ & $-1.872^{\star}$ & 1.078 & 0.136 & -0.636 & -0.350 & $-1.661^{\star}$ & & & \\
\hline$D \times C$ & -0.736 & 1.608 & 0.829 & $-2.523^{\star \star}$ & -0.919 & 0.308 & 1.087 & & \\
\hline $\mathrm{O} \times \mathrm{C}$ & $2.097^{\star}$ & -0.008 & 0.435 & 1.043 & 1.239 & 1.461 & 0.122 & 0.092 & \\
\hline$V \times C$ & 0.950 & $1.890^{\star}$ & 1.393 & $-2.017^{\star}$ & 0.178 & 1.648 & 1.443 & 0.640 & -0.405 \\
\hline
\end{tabular}

Overall, the average performance over all conditions; $A \times C$, alerting by conflict; $O \times C$, orienting by conflict; $V \times C$, validity by conflict. ${ }^{*} p<0.05,{ }^{* *} p<0.01$.

TABLE 8 | Correlation coefficients for the attentional effects in different groups between the ANT-V and the ANT-A.

\begin{tabular}{|c|c|c|c|c|c|c|c|c|c|c|}
\hline & Overall & Alerting & Disengaging & Orienting & Validity & Conflict & $A \times C$ & $D \times C$ & $O \times C$ & $V \times C$ \\
\hline AVGP & $0.392^{*}$ & $0.396^{\star}$ & 0.110 & 0.146 & 0.233 & 0.195 & 0.056 & 0.022 & -0.036 & 0.138 \\
\hline NAVGP & $0.417^{\star}$ & -0.339 & 0.157 & 0.237 & -0.090 & -0.051 & -0.144 & -0.117 & 0.135 & -0.060 \\
\hline$z$ & -0.109 & 2.805 & -0.174 & -0.344 & 1.191 & 0.903 & 0.731 & 0.507 & -0.625 & 0.723 \\
\hline$p$ & 0.457 & 0.003 & 0.431 & 0.366 & 0.117 & 0.183 & 0.232 & 0.306 & 0.266 & 0.235 \\
\hline
\end{tabular}

Overall, the average performance over all conditions; $A \times C$, alerting by conflict; $O \times C$, orienting by conflict; $V \times C$, validity by conflict. ${ }^{*} p<0.05$.

indicating that a supramodal alerting function that operates independently of sensory modality can be influenced by the experience of AVGs.

The significant interaction in RTs between orienting and conflict in the AVGP group suggests that AVGs directly benefit orienting to executive control. The marginally significant interaction in RTs between disengagement and conflict in the NAVGP group may suggest that $\mathrm{nAVG}$ may reduce the ability to disengage to executive control. When the conflicting stimuli occurs, AVGPs can quickly focus their attention on the target while NAVGPs may not be good at disengaging their attention from task-irrelevant distractors (Callejas et al., 2005). Consistent with the findings of Dye et al. (2009) and Gao et al. (2018), the present results indicate that the experience of AVG is associated with enhanced guidance of the target and inhibition of distractors. As frequent players of AVGs, AVGPs can rapidly allocate attention to the informative stimulus (orienting) and disengage attention from irrelevant stimuli (disengagement) and then employ the engagement of attentional resources to detect and resolve the conflict embedded in a complex game environment. It is noteworthy that the advantage from AVGs was found in both visual and auditory tasks, indicating a general common influence of AVGs on attentional functions, irrespective of visual or auditory modalities.

The significant correlation between RTs for visual and auditory tasks in the alerting function for AVGPs indicates that a supramodal, irrespective of specific modality, alerting effect can be influenced by AVGs. Inconsistent with results of the present study, by examining the ordinary people, Spagna et al. (2015) found that alerting relies on modality-specific processes, which can be explained in that the alerting effect requires no active reaction in the auditory modality but requires attention for turning toward targets in the visual modality (Posner, 1978).
However, after the experience of playing AVGs, the modalityspecific alerting function becomes supramodal and can operate independently of modality, suggesting a more sensitive state of readiness for multiple inputs to deal with the upcoming challenges in a game. Donohue et al. (2010) also reported that AVGPs can distinguish visual and auditory stimuli that occurs at the same moment or are slightly offset in time, revealing an enhanced alerting ability in both modalities. Since the enemies in AVGs are often recognized by motion or luminance transients, the alerting function influenced by playing AVGs may reveal the plastic bottom-up attention mechanism.

There were more correlations among RTs for the three attentional effects in the AVGPs than in the NAVGPs in the auditory task, suggesting that AVGs are associated with better attentional skills (Green and Bavelier, 2003). The correlation between alerting and conflict was stronger for AVGPs, while the correlations for other functions were tighter for NAVGPs in the visual task, suggesting that AVGs benefit abilities associated with alerting and executive control. AVG playing has been found to be associated with neural changes in the prefrontal cortex (Moisala et al., 2017), left dorsolateral prefrontal cortex and frontal eye fields (Kuhn et al., 2014). This suggests the relationship between AVGs and executive control, which refers to the higher-level ability to focus on the task and ignore noise over space and time (Green and Bavelier, 2015). Notably, the results of the present study showed that AVGPs not only heightened correlations for the executive control effect, which is likely associated with top-down processes (Fan, 2014), but also facilitated more connections with the alerting that exists as an exogenous form of attention, which is considered to be mediated by bottom-up processes (Fan et al., 2002). The results indicate the effects of AVGs on both top-down and bottom-up processing of attention. 
There were some limitations to the present study. First, the impact of AVGs on the attentional functions suggests directionality. As the attentional functions of participants before they started to play AVG are unclear, the possibility of attentional functions having an effect on AVG playing cannot be completely ruled out. Future research can utilize a training design to explore the directionality issue (Green and Bavelier, 2007, 2015; Bavelier and Green, 2019). In the training design, participants are randomly assigned to play AVGs and the impact of AVGs can be assessed by comparing the cognitive measures taken before and after training. Second, although Feng et al. (2007) found that playing AVGs can reduce gender differences in spatial attention and spatial cognition, the effect of AVGs on different attentional functions remains unclear. To control for the possible effect of imbalanced gender ratio between groups, we included gender as a covariate in our analyses (i.e., ANCOVA), and the effects of demographic variables in groups differing in gaming experience should be further investigated.

\section{CONCLUSION}

The results of the present study confirmed the benefits of AVGs for attentional functions, especially for the interaction between orienting and executive control, and the supramodal alerting function. Complementing research on the benefits of AVGs in other domains, such as motivation (Ventura et al., 2013), emotion (Aldao et al., 2010) and social behavior (Gill, 2012), the findings of the present study provide insight into the potential positive implications of video games. As some studies have investigated the effects of various types of video games (Dobrowolski et al., 2015) and the neural mechanisms underlying different attentional functions in ordinary people (Liu et al.,

\section{REFERENCES}

Aldao, A., Nolen-Hoeksema, S., and Schweizer, S. (2010). Emotion-regulation strategies across psychopathology: A meta-analytic review. Clin. Psychol. Rev. 30, 217-237. doi: 10.1016/j.cpr.2009.11.004

Anderson, C. A., Shibuya, A., Ihori, N., Swing, E. L., Bushman, B. J., Sakamoto, A., et al. (2010). Violent video game effects on aggression, empathy, and prosocial behavior in Eastern and Western countries: A meta-analytic review. Psychol. Bull. 136, 151-173. doi: 10.1037/a0018251

Bailey, K., West, R., and Anderson, C. (2010). A negative association between video game experience and proactive cognitive control. Psychophysiology 47, 34-42. doi: 10.1111/j.1469-8986.2009.00925.x

Bavelier, D., Achtman, R. L., Mani, M., and Föcker, J. (2012). Neural bases of selective attention in action video game players. Vis. Res. 61, 132-143. doi: 10.1016/j.visres.2011.08.007

Bavelier, D., and Green, C. S. (2019). Enhancing Attentional Control: Lessons from Action Video Games. Neuron 104, 147-163. doi: 10.1016/j.neuron.2019. 09.031

Callejas, A., Lupiàñez, J., Funes, M. J., and Tudela, P. (2005). Modulations among the alerting, orienting and executive control networks. Exp. Brain Res. 167, 27-37. doi: 10.1007/s00221-005-2365-z

Dobrowolski, P., Hanusz, K., Sobczyk, B., Skorko, M., and Wiatrow, A. (2015). Cognitive enhancement in video game players: The role of video game genre. Comput. Hum. Behav. 44, 59-63. doi: 10.1016/j.chb.2014.11.051

Donohue, S. E., Woldorff, M. G., and Mitroff, S. R. (2010). Video game players show more precise multisensory temporal processing abilities. Attention 72, 1120-1129. doi: 10.3758/app.72.4.1120
2010), future research employing brain imaging techniques is required to explore the different effects of varied games on the neural mechanisms of attentional functions.

\section{DATA AVAILABILITY STATEMENT}

The raw data supporting the conclusions of this article will be made available by the authors, without undue reservation.

\section{ETHICS STATEMENT}

The studies involving human participants were reviewed and approved by the Ethics Committee of Tianjin Normal University. The patients/participants provided their written informed consent to participate in this study.

\section{AUTHOR CONTRIBUTIONS}

XW and YiJ conceived the study. YiJ analyzed the data. All authors discussed the results and contributed to the writing of the manuscript.

\section{FUNDING}

This work was supported by the National Natural Science Foundation of China under Grant (No. 31700938), Tianjin Municipal Education Commission (No. 2019SK056), and Doctoral research project of Tianjin Normal University (No. 52WW2102).

Dye, M. W. G., Green, C. S., and Bavelier, D. (2009). The development of attention skills in action video game players. Neuropsychologia 47, 1780-1789. doi: 10 . 1016/j.neuropsychologia.2009.02.002

Fan, J. (2014). An information theory account of cognitive control. Front. Hum. Neurosci. 8:680.

Fan, J., Gu, X., Guise, K. G., Liu, X., Fossella, J., Wang, H., et al. (2009). Testing the behavioral interaction and integration of attentional networks. Brain Cogn. 70, 209-220. doi: 10.1016/j.bandc.2009.02.002

Fan, J., Hof, P. R., Guise, K. G., Fossella, J. A., and Posner, M. I. (2008). The functional integration of the anterior cingulate cortex during conflict processing. Cereb. Cortex 18, 796-805. doi: 10.1093/cercor/bhm125

Fan, J., Mccandliss, B. D., Sommer, T., Raz, A., and Posner, M. I. (2002). Testing the efficiency and independence of attentional networks. J. Cogn. Neurosci. 14, 340-347. doi: 10.1162/089892902317361886

Feng, J., Spence, I., and Pratt, J. (2007). Playing an action video game reduces gender differences in spatial cognition. Psychol. Sci. 18, 850-855. doi: 10.1111/ j.1467-9280.2007.01990.x

Franceschini, S., Trevisan, P., Ronconi, L., Bertoni, S., and Gori, S. (2017). Action video games improve reading abilities and visual-to-auditory attentional shifting in english-speaking children with dyslexia. Scientific Rep. 7:5863.

Gao, Y. L., Zhang, S. H., Zhang, Y., Wang, M. X., Li, Y. X., Wang, Z. N., et al. (2018). Action Video Games Influence on Audiovisual Integration in Visual Selective Attention Condition. DEStech Transact. Biol. Health. 2018, 248-254.

Gill, N. (2012). 10 most popular Facebook games in 2012 - Popular apps!!. URL: http://www.socialdon.com/blog/popularfacebook-games-2012/

Granic, I., Lobel, A., Rutger, C. M. E., and Engels. (2014). The Benefits of Playing Video Games. Am. Psychol. 69, 66-78. 
Green, C. S., and Bavelier, D. (2003). Action video game modifies visual selective attention. Nature 423, 534-537. doi: 10.1038/nature01647

Green, C. S., and Bavelier, D. (2007). Action-video-game experience alters the spatial resolution of vision: Research article. Psychol. Sci. 18, 88-94. doi: 10. 1111/j.1467-9280.2007.01853.x

Green, C. S., and Bavelier, D. (2015). Action video game training for cognitive enhancement. Curr. Opin. Behav. Sci. 4, 103-108. doi: 10.1016/j.cobeha.2015. 04.012

Green, C. S., Pouget, A., and Ba Velier, D. (2010). Improved probabilistic inference as a general learning mechanism with action video games. Curr. Biol. 20, 1573-1579. doi: 10.1016/j.cub.2010.07.040

Hubert-Wallander, B., Green, C. S., Sugarman, M., and Bavelier, D. (2011). Changes in search rate but not in the dynamics of exogenous attention in action videogame players. Attention 73, 2399-2412. doi: 10.3758/s13414-011-0194-7

Kuhn, S., Lorenz, R., Banaschewski, T., Barker, G. J., Buchel, C., Conrod, P. J., et al. (2014). Positive association of video game playing with left frontal cortical thickness in adolescents. PLoS One 9:e91506. doi: 10.1371/journal. pone.0091506

Krishnan, L., Kang, A., Sperling, G., and Srinivasan, R. (2013). Neural Strategies for Selective Attention Distinguish Fast-Action Video Game Players. Brain Topogr. 26, 83-97. doi: 10.1007/s10548-0120232-3

Li, M., and Tsai, C. (2013). Game-Based Learning in Science Education: A Review of Relevant Research. J. Sci. Educat. Technol. 22, 877-898. doi: 10.1007/s10956013-9436-x

Liu, X., Park, Y., Gu, X. S., and Fan, J. (2010). Dimensional overlap accounts for independence and integration of stimulus-Response compatibility effects. Attention 72, 1710-1720. doi: 10.3758/app.72.6. 1710

Moisala, M., Salmela, V., Hietajärvi, L., Carlson, S., Vuontela, V., Lonka, K., et al. (2017). Gaming is related to enhanced working memory performance and taskrelated cortical activity. Brain Res. 1655, 204-215. doi: 10.1016/j.brainres.2016. 10.027

Posner, M. I. (1978). Chronometric exploration of mind, O. U. Press Edn. New York: Lawrence Erlbaum, 271.
Prescott, A. T., Sargent, J. D., and Hull, J. G. (2018). Metaanalysis of the relationship between violent video game play and physical aggression over time. Proc. Natl. Acad. Sci. 115, 9882-9888. doi: 10.1073/pnas.1611617114

Sandro, F., and Sara, B. (2018). Improving Action Video Games Abilities Increases the Phonological Decoding Speed and Phonological Short-Term Memory in Children with Developmental Dyslexia. Neuropsychologia 130, 100-106. doi: 10.1016/j.neuropsychologia.2018.10.023

Spagna, A., Mackie, M. A., and Fan, J. (2015). Supramodal executive control of attention. Front. Psychol. 6, 1-14.

Spagna, A., He, G. X., Jin, S. C., Gao, L. L., Mackie, M. A., Tian, Y. H., et al. (2018). Deficit of supramodal executive control of attention in schizophrenia. J. Psychiatric Res. 97, 22-29. doi: 10.1016/j.jpsychires.2017.11.002

Spagna, A., Wu, T. T., Kim, K., and Fan, J. (2020). Supramodal executive control of attention: evidence from unimodal and crossmodal dual conflict effects. Cortex 133, 266-276. doi: 10.1016/j.cortex.2020.09.018

Ventura, M., Shute, V., and Zhao, W. (2013). The relationship between video game use and a performance-based measure of persistence. Comput. Educat. 60, 52-58. doi: 10.1016/j.compedu.2012.07.003

West, R., Swing, E. L., Anderson, C. A., and Prot, S. (2020). The contrasting effects of an action video game on visuo-spatial processing and proactive cognitive control. Int. J. Environ. Res. Public Health 17:5160. doi: 10.3390/ijerph1714 5160

Xiang, M. Q., and Hu, G. D. (2010). Application of Action Video Games in Visual Attention Skill Training. Chin. J. Clin. Psychol. 18, 390-392.

Conflict of Interest: The authors declare that the research was conducted in the absence of any commercial or financial relationships that could be construed as a potential conflict of interest.

Copyright (C) 2021 Wu, Jiang, Jiang, Chen, Chen and Bai. This is an open-access article distributed under the terms of the Creative Commons Attribution License (CC BY). The use, distribution or reproduction in other forums is permitted, provided the original author(s) and the copyright owner(s) are credited and that the original publication in this journal is cited, in accordance with accepted academic practice. No use, distribution or reproduction is permitted which does not comply with these terms. 